\title{
Quantitative Sensory Testing and Current Perception Threshold Testing in Patients With Chronic Pain Following Lower Extremity Fracture
}

Biological Research for Nursing 2018, Vol. 20(I) 16-24 (C) The Author(s) 2017 Reprints and permission: sagepub.com/journalsPermissions.nav DOI: 10.1177/1099800417720725 journals.sagepub.com/home/brn (S)AGE

\author{
Mari A. Griffioen, PhD, RN', Joel D. Greenspan, $\mathbf{P h D}^{2}$, \\ Meg Johantgen, PhD, RN ${ }^{3}$, Kathryn Von Rueden, MS, RN, CNS-BC, FCCM ${ }^{3}$, \\ Robert V. O'Toole, MD ${ }^{4}$, Susan G. Dorsey, PhD, RN, FAAN ${ }^{3}$, \\ and Cynthia L. Renn, PhD, RN ${ }^{3}$
}

\begin{abstract}
Background: Chronic pain is a significant problem for patients with lower extremity injuries. While pain hypersensitivity has been identified in many chronic pain conditions, it is not known whether patients with chronic pain following lower extremity fracture report pain hypersensitivity in the injured leg. Purpose: To quantify and compare peripheral somatosensory function and sensory nerve activation thresholds in persons with chronic pain following lower extremity fractures with a cohort of persons with no history of lower extremity fractures. Method: This was a cross-sectional study where quantitative sensory testing and current perception threshold testing were conducted on the injured and noninjured legs of cases and both legs of controls. Results: $A$ total of 14 cases and 28 controls participated in the study. Mean time since injury at the time of testing for cases was 22.3 (standard deviation $=12.1$ ) months. The warmth detection threshold $(p=.024)$ and nerve activation thresholds at $2,000 \mathrm{~Hz}(p<.00 \mathrm{I})$ and $250 \mathrm{~Hz}(p=.002)$, respectively, were significantly higher in cases compared to controls. Conclusion: This study suggests that patients with chronic pain following lower extremity fractures may experience hypoesthesia in the injured leg, which contrasts with the finding of hyperesthesia previously observed in other chronic pain conditions but is in accord with patients with nerve injuries and surgeries. This is the first study to examine peripheral sensory nerve function at the site of injury in patients with chronic pain following lower extremity fractures using quantitative sensory testing and current perception threshold testing.
\end{abstract}

\section{Keywords}

chronic pain, traumatic lower extremity fracture, quantitative sensory testing, current perception threshold testing

Chronic pain is a significant problem for patients with traumatic injuries (Jenewein et al., 2009; Williamson, Gabbe, Cameron, Edwards, \& Richardson, 2009), especially for those with injuries to the lower extremities (Rivara et al., 2008; Williamson et al., 2009). The consequences of chronic pain are substantial, as individuals with trauma-related chronic pain miss more days of work and seek medical care more frequently than those without chronic pain (Jenewein et al., 2009). In addition, persons with posttrauma chronic pain report high levels of pain intensity, anxiety, and depression (Stålnacke, 2011), and $87 \%$ state that pain interferes with their daily activities (Clay et al., 2010). Risk factors for chronic pain in trauma patients include older age, untreated anxiety and/or depression, female gender, fewer years of education, and high pain intensity at the time of hospital admission (Clay, Watson, Newstead, \& McClure, 2012; Rivara et al., 2008; Rosenbloom, Khan, McCartney, \& Katz, 2013); however, the underlying physiological mechanisms associated with chronic pain following injury remain unclear.

During the acute phase of injury, sensitization develops, so that subsequent stimuli are intensified, which leads to guarding at the site of injury to prevent further damage. This heightened peripheral sensitivity generally returns to baseline as the injury heals (Latremoliere \& Woolf, 2009).

\footnotetext{
I School of Nursing, University of Delaware, Newark, DE, USA

${ }^{2}$ School of Dentistry, University of Maryland, Baltimore, MD, USA

${ }^{3}$ School of Nursing, University of Maryland, Baltimore, MD, USA

${ }^{4}$ School of Medicine, University of Maryland, Baltimore, MD, USA
}

\section{Corresponding Author:}

Mari A. Griffioen, PhD, RN, School of Nursing, University of Delaware, $25 \mathrm{~N}$. College Avenue, Newark, DE 19716, USA.

Email: mgriffi@udel.edu 
Table I. Inclusion and Exclusion Criteria for the Lower Extremity Fracture (With Chronic Pain) and No-Fracture (Healthy Control) Groups.

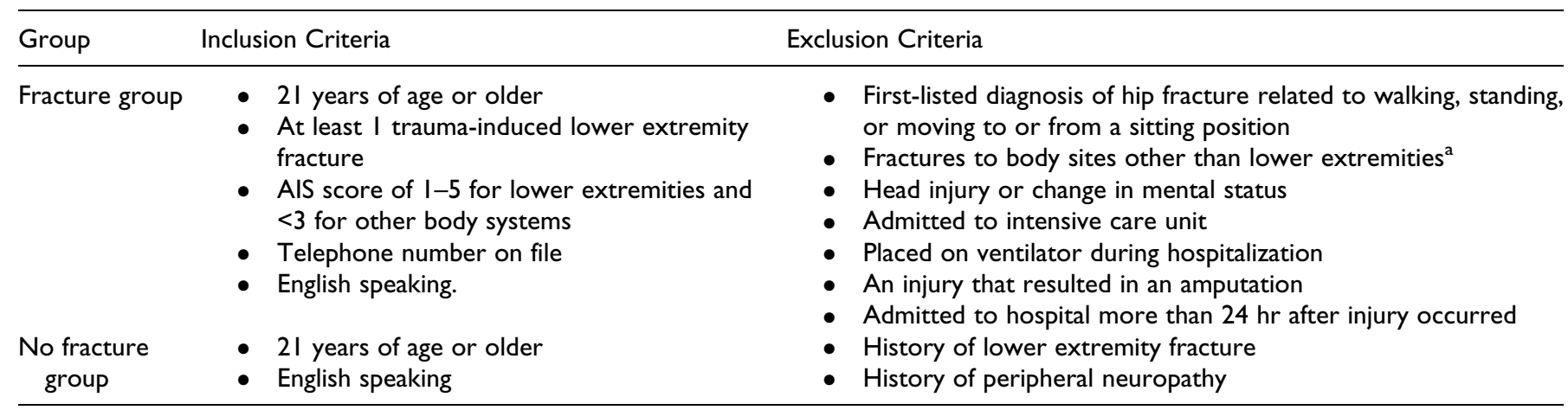

Note. AIS = Abbreviated Injury Scale.

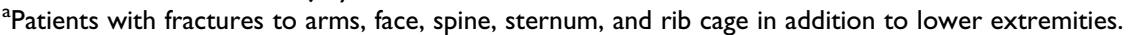

Researchers have identified phenomena such as pain hypersensitivity with associated changes in the nociceptive pathways in patients with chronic pain (Woolf, 2011), but whether increased pain sensitivity persists past the acute phase in patients with lower extremity fracture and contributes to chronic pain remains unclear.

Quantitative sensory testing (QST) and current perception threshold (CPT) testing are two techniques that can be used to quantify pain hypersensitivity. QST refers to the use of a set of objective testing procedures that allow the examiner to measure and quantify somatosensory function in large (A $\beta$ fibers) and small sensory nerve fibers (A $\delta$ and $\mathrm{C}$ fibers; Rolke, Baron, et al., 2006). The aim is to detect sensory loss (i.e., hypoesthesia, hypoalgesia) or sensory gain (i.e., hyperaesthesia, hyperalgesia, allodynia) in large and small sensory nerve fibers (Rolke, Baron, et al., 2006). CPT noninvasively quantifies the functioning of both unmyelinated and myelinated sensory nerve fibers and determines the lowest level (threshold) of electrical current that triggers a painless sensation (i.e., sensory nerve activation threshold; Neurotron Inc., Innovative Medical Technology, Baltimore, MD). Large myelinated $\mathrm{A} \beta$ fibers are most sensitive to stimulation at $2,000 \mathrm{~Hz}$, small myelinated $\mathrm{A} \delta$ fibers at $250 \mathrm{~Hz}$, and small unmyelinated $\mathrm{C}$ fibers at $5 \mathrm{~Hz}$ (Rendell et al., 1989).

Nurses care for patients with chronic pain in a variety of practice settings, often engaging in patient education regarding a specific illness, disease, or symptom. Study findings regarding the underlying pathology and associated symptoms of chronic pain following lower extremity fracture will allow nurses to provide patient education regarding the specific sensory abnormality that may be present. Investigators have conducted little research to characterize peripheral sensory nerve function in patients with chronic pain following lower extremity fracture. The purpose of the present study was to use QST and CPT to record the responses of patients with chronic pain following lower extremity fracture and of healthy controls to innocuous and noxious mechanical and thermal stimuli. We hypothesized that patients would display symptoms of pain hypersensitivity in the fractured leg compared to the controls.

\section{Method}

\section{Design and Participants}

Following university and hospital institutional review board approval, we enrolled participants with chronic pain from a concurrent cross-sectional cohort study in which we were examining the association between acute pain characteristics and chronic pain following lower extremity fractures (Griffioen et al., 2017). For that study, we screened the trauma registry for patients who were admitted to a large academic urban trauma center between July 2011 and June 2014 and met the inclusion and exclusion criteria, as shown in Table 1. To be considered for the present study, patients had to present with at least one trauma-induced lower extremity fracture that had occurred at least 6 months prior to data collection. To minimize confounding from pain due to severe injuries in other body sites, investigators limited the Abbreviated Injury Scale (AIS) score to less than 3 for body systems other than the lower extremities. The total number of AIS scores a patient receives correlates with the number of injuries sustained (Trauma.org, 2007). To ensure a homogenous sample, we also excluded patients whose firstlisted diagnosis was hip fracture that occurred while walking, standing, or moving to or from a sitting position or who had fractures to other body sites in addition to the lower extremities, had a head injury, were admitted to the intensive care unit, had an injury that resulted in an amputation, and/or were admitted to the hospital more than 24 hours after the injury occurred. We contacted eligible English-speaking patients with a telephone number on file during normal business hours (9 a.m. to 5 p.m.). Following a description of the study, we invited all participants who consented to participate in the concurrent study to participate in the current study testing sensory nerve function. We gave interested participants an appointment for a study visit and obtained written consent at that time.

We enrolled a control group of healthy participants with no lower extremity fracture via advertisement at the university. We screened potential control participants over the phone and invited those meeting the study criteria of age of 21 years or older, ability to speak and read English, and no history of lower extremity fractures or diagnosis of peripheral neuropathy to 
participate. We did not exclude individuals from the control group if they had a history of other pain conditions.

The clinic visit for both patients and controls lasted approximately $3 \mathrm{hr}$, and all participants received a US\$75 gift card and a parking pass. Of the 516 eligible cases, 176 answered the phone and 34 were interested in participating in the study. The final study sample was 14 cases ( 20 were no-shows) and 28 controls.

\section{Study Measures}

Our primary outcome measures were participants' response to innocuous and noxious mechanical and thermal stimuli. To characterize the study sample, we asked participants to rate their current health on a scale from excellent to poor, whether they had ever been diagnosed with a chronic pain condition, and whether they were currently taking prescription or overthe-counter medication for pain. Variables we collected from medical records included age, gender, race, length of hospital stay, mechanism of injury, fracture site, AIS scores, and whether they had a surgical procedure for their injury. Further, participants completed two pain, one depression, and one anxiety questionnaire.

QST. QST consisted of recording participants' (1) mechanical detection thresholds for touch and vibration, (2) ability to differentiate between pinprick and blunt pressure, (3) thermal detection thresholds for perception of cold and warm stimuli, (4) thermal pain thresholds for cold and heat stimuli, (5) pressure pain threshold, and (6) CPT. We conducted all testing on both legs at the inner aspect of the calf centered between the knee and the ankle on normal skin (minimal scarring). Testing proceeded in the above referenced order following a standardized protocol. We asked participants to keep their eyes closed during mechanical vibration, pinprick, and pressure pain testing.

Mechanical detection threshold. We tested mechanical touch thresholds using a standard set of 20 Semmes-Weinstein monofilaments, which are calibrated to bend at a specified amount of force (0.008-300 g; www.bioseb.com). We applied the fibers, in ascending order by force calibration, perpendicular to the testing site until they just bent, held them in place for $1 \mathrm{~s}$ and removed them. We repeated each fiber application 3 times with a 1-s break between stimuli. We recorded the mechanical detection threshold the point at which the participant reported the ability to detect the fiber during all three applications

Pinprick detection. We completed pinprick detection using a sterile needle in the head of a Buck neurological hammer (sharp stimulus) and a sterile paper clip that had one end bent at $90^{\circ}$ away from the clip body to form a probe (dull stimulus). After each application of the stimulus, we asked the participant to identify whether the sensation was sharp or dull. We administered a total of six applications in a random order. The percentage of correct responses out of total responses was the pinprick detection threshold.
Vibration detection. We used a graduated tuning fork (RydelSeiffer; US Neurologicals, Poulsbo, WA) for vibration detection. We set the tuning fork to vibrate and placed it on the tibia midway between the knee and ankle with the markings facing away from participants. During the test, we asked participants to report when they no longer felt the vibration. We recorded the number on the calibrated weight that was nearest to the intersection of the triangles as the vibration detection threshold. We performed this test 3 times and calculated a mean score.

Thermal detection and pain thresholds. We conducted thermal threshold testing using the Medoc Pathway Pain and Sensory Evaluation System (Medoc, Ramat Yishai, Israel). For the test, we placed a thermode with a $9 \mathrm{~cm}^{2}$ surface area on the test site. We set the baseline temperature of the thermode to $32^{\circ} \mathrm{C}$ and increased or decreased the temperature from baseline with a $1^{\circ} \mathrm{C} / \mathrm{s}$ ramp. For safety, we programmed automatic cutoff temperatures of $0^{\circ} \mathrm{C}$ and $50^{\circ} \mathrm{C}$ into the thermal stimulator. We instructed the participant to press the stop button at the first sensation of cold, cold pain, warmth, heat pain, and maximum tolerable heat pain. We calculated the mean detection threshold temperatures from three consecutive measurements.

Pressure pain threshold. To determine the pressure pain threshold, we placed the Medoc Pressure Algometer (Medoc) application surface (flat rubber tip $1 \mathrm{~cm}^{2}$ ) on the test site. The device increased pressure at a steady rate $(0.3 \mathrm{kgF} / \mathrm{s})$ until the participant pressed the button when the pressure became painful or the stimulus reached $6.11 \mathrm{kgF}(600 \mathrm{kPa})$. We administered five trials and recorded the mean of the two values that were closest to each other as the threshold estimate.

CPT. We used a standardized automated double-blind testing methodology for CPT testing. We coated a pair of $1-\mathrm{cm}$ gold electrodes, separated by $1.7 \mathrm{~cm}$, with a thin layer of conductive electrode gel and taped them to the test site. Before the actual test, we conducted an automated intensity alignment procedure to narrow down the range of possible measures. We instructed participants to press a button once they felt a stimulus at the site of the electrode. Testing proceeded at 2,000, 250, and $5 \mathrm{~Hz}$. The device presented the participant with randomly generated pairs of real and placebo (no current) stimuli, and we asked the participant to identify which of the two stimuli was real. Based on the response, the CPT device adjusted the output level of the stimulus current and randomly generated a new testing order for the next pair of tests in the series. Physiological definition of the exact threshold level was the lowest stimulus that a participant was able to perceive $50 \%$ of the time.

Chronic pain. We used the Chronic Pain Grading Scale (CPGS) to assess for chronic pain. The CPGS measures chronic pain as a function of pain intensity and pain-related disability (Korff, Ormel, \& Dworkin, 1992). The characteristic pain intensity score is the sum of the current, worst, and average pain rated on an 11-point Likert-type scale with a range from 0 to 30 . We categorized participants as having chronic pain related to the lower extremity fracture when the characteristic pain intensity 
score ranged from 1 to 30 . We set a threshold of 1 as our definition of chronic pain as researchers have used this definition in prior studies (Castillo, MacKenzie, Wegener, \& Bosse, 2006; Rivara et al., 2008). We also examined the number of days during which participants reported experiencing pain over the previous 6 months. For the disability score, participants reported the number of days they had been unable to do usual activities over the previous 6 months and rated on a scale from 0 to 10 how much the pain interfered with daily, recreational/ social/family, and work activities over that period (range: $0-40$ ). Research has shown the CPGS to be a valid and reliable instrument (Cronbach's $\alpha=.74$ ) for the assessment of chronic pain (Elliott, Smith, Smith, \& Chambers, 2000; Korff et al., 1992), and investigators have used it to assess for pain in patients with traumatic injuries (Castillo et al., 2006; Rivara et al., 2008). We asked participants in the fracture group to focus on pain related to the site of injury and those in the no-fracture group on pain at any body site.

Quality of pain. To describe the quality of pain experienced by participants, we administered the short-form McGill Pain Questionnaire-2 (SF-MPQ-2). The SF-MPQ-2 measures the qualities of neuropathic and nonneuropathic pain felt during the preceding week (Dworkin et al., 2009). The 22 items measure four dimensions of pain: (1) continuous pain, (2) intermittent pain, (3) neuropathic pain, and (4) affective descriptors. Respondents score each descriptor on a numeric rating scale from 0 to 10 . The total score is a mean of all SF-MPQ-2 item ratings, with lower scores indicating a lower level of pain. We also calculated mean scores for each dimension. To determine the type of pain most frequently reported by a participant, we calculated the frequency of each descriptor. The SF-MPQ-2 has good internal consistency (Cronbach's $\alpha=.96$; Lovejoy, Turk, $\&$ Morasco, 2012) and reliability (Cronbach's $\alpha=.91$; Dworkin et al., 2009), and researchers have used it to assess for chronic pain following traumatic injury (Clay et al., 2010).

Depression. We administered the Beck Depression Inventory, second edition (BDI-II) to determine depressive symptoms and severity at the time of testing. It includes items measuring cognitive, affective, somatic, and vegetative symptoms of depression and has a recall period of 2 weeks (Smarr \& Keefer, 2011). The BDI-II contains 21 items, with each item rated on a 4-point scale ranging from 0 (not at all) to 3 (extreme). The depression score is the sum of the scores on the 21 items, and total scores range from 0 to 63 . The BDI-II has high internal consistency (Cronbach's $\alpha=.92$ ) in patients with chronic pain (Harris \& D’Eon, 2008).

Anxiety. So that we could evaluate participants' current state of anxiety (subjective feelings of apprehension, tension, nervousness, worry, and activation/arousal of the autonomic nervous system) and trait of anxiety (calmness, confidence, and security), the participants completed the State-Trait Anxiety Inventory (STAI; Julian, 2011). The 20 items of the state anxiety section, which assess the intensity of feelings at the current moment, ask respondents to select one of the four possible responses: $1=$ not at all, $2=$ somewhat, $3=$ moderately so, and $4=$ very much so. Likewise, for the 20 items of the trait anxiety section, which assess general feelings, the respondent chooses among the following four options: $1=$ almost never, $2=$ sometimes, $3=$ often, or $4=$ almost always. Item scores are summed to obtain subtest total scores, which range from 20 to 80 for each subtest. A higher score indicates higher levels of anxiety. Internal consistency (Cronbach's $\alpha=.95$ ) and validity are high $(r=.85)$. Researchers have used the STAI to evaluate anxiety in patients with chronic pain (Newcomer, Shelerud, Douglas, Larson, \& Crawford, 2010).

\section{Data Analysis}

We conducted analyses using SPSS 22.0 (IBM SPSS Statistics for Windows, Version 22.0; IBM Corp, Armonk, NY). Summary statistics included the mean, standard deviation $(S D)$, and range for continuous variables and frequencies and proportions for categorical variables. We used Mann-Whitney $U$ test, Wilcoxon signed rank test, $\chi^{2}$ test of independence, and Fisher's exact to calculate group differences.

\section{Results \\ Participant Characteristics}

Characteristics of participants with and without fractures are detailed in Table 2 . We found statistically significant differences between the fracture and no-fracture groups in current health rating, currently taking pain medication, and depression. The mean time since injury at the time of testing for the fracture group was 22.3 months $(S D=12.1$, range $=10-30)$. Almost all participants with fractures $(86 \%)$ had experienced fibula/tibia fractures, with the next most frequent being fractures in the ankle and pelvis.

All participants with fractures reported having pain at the site of injury at the time of testing; $64 \%$ reported experiencing pain every day, and the range of number of days with pain for those with intermittent pain was $7-170$ days $(7,72,90,96$, 170 days). The mean pain intensity score at the time of testing was $3.7(S D=2.2)$, the worst pain in the last 3 months was 7.7 $(S D=2.0)$, and the average pain was $4.8(S D=1.9)$. The mean characteristic pain intensity score from the CPGS was 16.2 $(S D=5.0)$, with the lowest score reported by an individual patient being 7 and the highest 25 . The mean disability score was $17.6(S D=9.9)$. Of participants in the no-fracture group, five reported having pain at the time of testing $(M=1.6, S D=$ 3.6). Some participants in the fracture $(n=4)$ group reported experiencing chronic pain of a different origin than the lower extremity fracture injury, while two participants with no-fracture also reported a history of chronic pain.

Total mean pain quality scores on the SF-MPQ-2 for the week prior to testing differed significantly between the fracture $(M=4.3, S D=1.5)$ and no-fracture groups, $M=0.7, S D=1.1$, $t(41)=-9.11, p<.001$. The lowest mean score in the fracture group was for affective descriptors $(M=3.1, S D=2.9)$, while 
Table 2. Participant Characteristics.

\begin{tabular}{|c|c|c|c|c|}
\hline Characteristic & No-Fracture Group $(n=28)$ & Fracture Group $(n=14)$ & $\chi^{2 / z}$ & $p$ \\
\hline Male, $n(\%)$ & $13(46)$ & II (79) & $2.734^{\mathrm{a}}$ & .098 \\
\hline White & $12(43)$ & $8(57)$ & & \\
\hline African American & $6(21)$ & $5(36)$ & & \\
\hline Other & $10(36)$ & I (7) & & \\
\hline Fair/poor & - & $2(14)$ & & \\
\hline History of chronic pain not injury related, $n(\%)$ & $2(7)$ & $4(29)$ & & .155 \\
\hline Take medication for pain, $n(\%)$ & 0 & $4(29)$ & & .009 \\
\hline Depressive symptoms, score on BDI-II, $M(S D)$ & $1.5(1.9)$ & $8.8(6.7)$ & -4.03 & $<.001$ \\
\hline \multicolumn{5}{|l|}{ STAI, $M(S D)$} \\
\hline State anxiety & $25.0(6.1)$ & $31.3(12.7)$ & -1.76 & .097 \\
\hline
\end{tabular}

Note. $N=42$. BDI-II = Beck Depression Inventory, second edition; SD = standard deviation; STAI = State-Trait Anxiety Inventory; $\chi^{2}=$ chi-square test; $z=$ Mann-Whitney $U$ test.

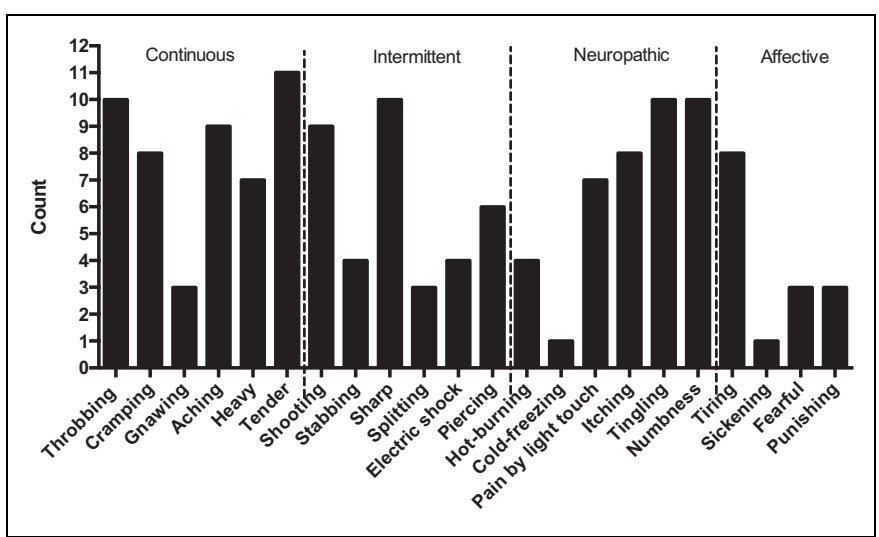

Figure I. Frequency with which participants in the fracture group $(n=14)$ chose each pain descriptor on the short-form McGill Pain Questionnaire-2 (SF-MPQ-2).

the highest was for continuous descriptors $(M=4.2, S D=2.1)$. The fracture group most frequently chose the descriptor tenderness, followed by throbbing, sharpness, tingling, and numbness (Figure 1). They reported the highest intensity for aching ( $M=$ 4.6, $S D=3.6)$ followed by throbbing $(M=3.7, S D=3.0)$ and shooting $(M=3.4, S D=2.9)$.

\section{QST}

The QST results for mechanical detection thresholds were similar for the fracture and no-fracture groups for touch, vibration, pinprick, and pressure pain as well as for the injured and noninjured legs in cases and right and left legs for controls. For thermal detection and pain threshold, one participant with a fracture did not record a response for either cold or warm temperatures and was excluded from the analysis. There were no differences in cold sensation, cold pain, heat pain, or heat tolerance between the fracture and no-fracture groups, the

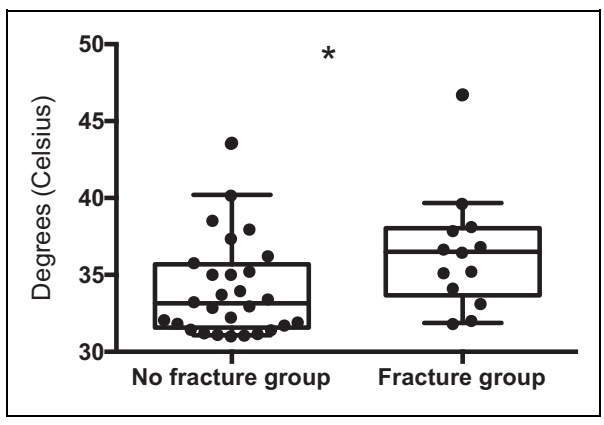

Figure 2. Warmth detection thresholds in the fracture group $(n=$ 13) versus the no-fracture group $(n=28)$. The box plot with Tukey's whiskers shows a significantly higher threshold in cases compared to controls. ${ }^{*} p<.05$. The whiskers represent the maximum and minimum values, excluding outliers.

injured and noninjured legs in participants with fractures, or the right and left legs in participants with no fractures. The median warmth detection threshold was significantly higher in the fracture group $\left(36.5^{\circ} \mathrm{C}, n=13\right)$ compared to the no-fracture group $\left(33.2^{\circ} \mathrm{C}, n=28\right), U=101.5, z=-2.256$, $p=0.024$ (Figure 2).

\section{CPT}

During CPT testing, the neurometer indicated inconsistent responses for five participants with fractures at $2,000 \mathrm{~Hz}$, for two at $250 \mathrm{~Hz}$, and for two at $5 \mathrm{~Hz}$ and for one participant with no fracture at 2,000 $\mathrm{Hz}$. In addition, one participant with fracture had inconsistent responses at all three frequencies. For another participant with fracture, no responses were recorded during CPT, which may be attributable to inadequate contact between the electrode and the skin, which can occur as a result of excessive tissue resistance due to skin changes (Neurotron Inc., Innovative Medical Technology). The final number of participants from the fracture group whose data were included 


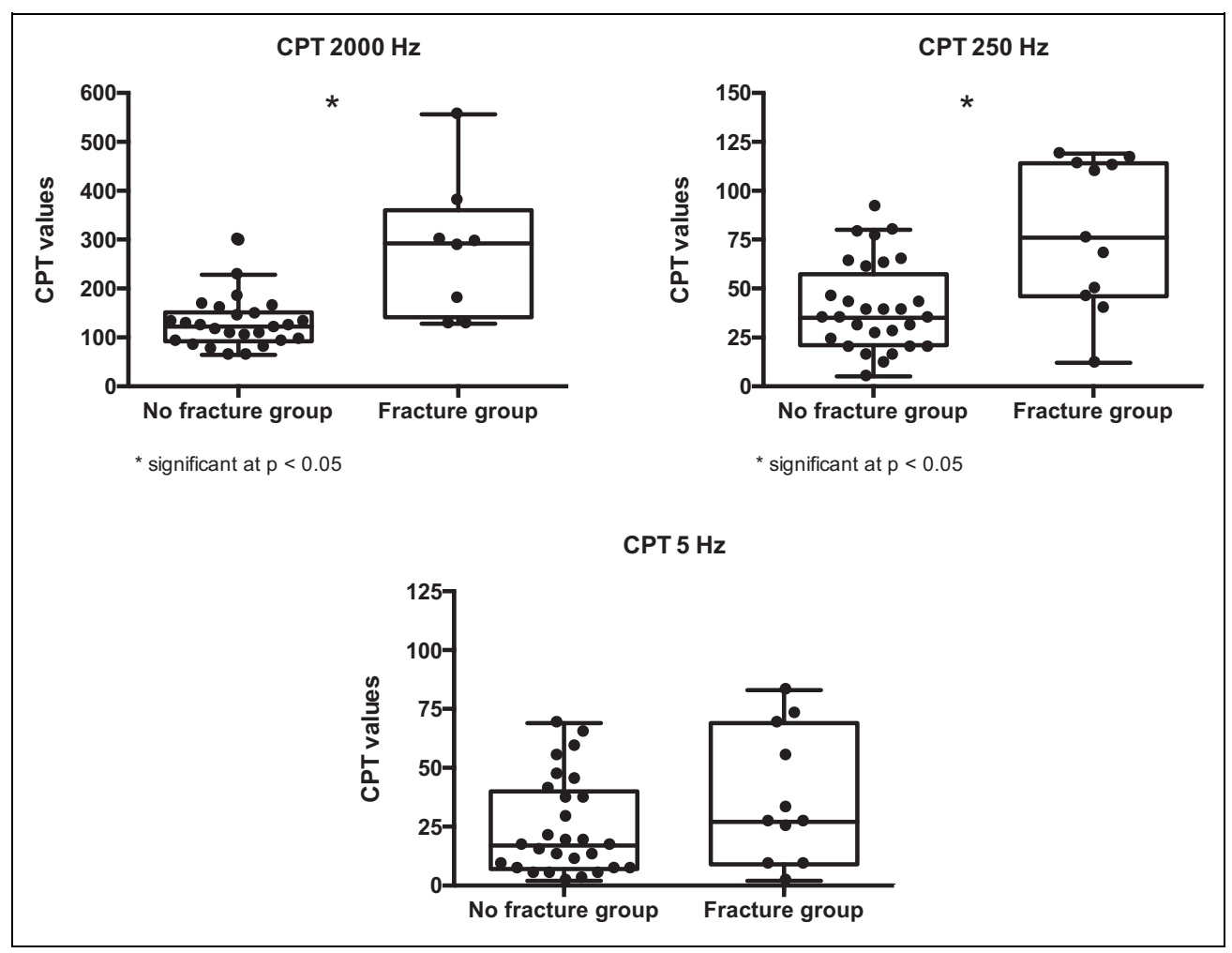

Figure 3. Current perception threshold (CPT) in the fracture versus the no-fracture group. Box plots with Tukey's whiskers show significant differences between cases and control at frequencies of 2,000 and $250 \mathrm{~Hz}$ but not $5 \mathrm{~Hz}$. $* p<.05$. The whiskers represent the maximum and minimum values, excluding outliers.

in the analyses was 8 at $2,000 \mathrm{~Hz}$ and 11 each at $250 \mathrm{~Hz}$ and $5 \mathrm{~Hz}$. We noted significant differences in median sensory nerve activation thresholds between the two groups at $2,000 \mathrm{~Hz}$ (fracture group: median $=292, n=8$; no-fracture group: median $=122, n=27 ; U=26.5 ; z=3.15, p<.001)$ and at $250 \mathrm{~Hz}$ (fracture group: median $=76, n=11$; no-fracture group: median $=35, n=28 ; U=57.0 ; z=3.03, p=0.002$; Figure 3 ). In addition, we found a significant difference between the injured and noninjured leg in participant with fracture at $250 \mathrm{~Hz}(z=-2.046, p=.41)$ but no significant difference between the right and left legs in participants with no fractures.

\section{Discussion}

In the present study, we compared response to innocuous and noxious mechanical and thermal stimuli using QST and CPT between patients with chronic pain following traumatic lower extremity fractures and a group of healthy controls with no fractures. The fracture group displayed significantly higher warmth detection thresholds and increased CPT measures for $\mathrm{A} \beta$ and $\mathrm{A} \delta$ fibers. In contrast to our hypothesis of pain hypersensitivity, these results suggest hypoesthetic sensory nerve function in the previously fractured leg of patients with chronic pain.

Research has found an increased warmth detection threshold in a range of chronic pain conditions such as traumatic partial nerve injuries (Leffler \& Hansson, 2008), persistent low back pain (Starkweather et al., 2016), knee pain (Jensen, Hystad, Kvale, \& Baerheim, 2007), and whiplash injuries (Raak \& Wallin, 2006). With traumatic injury, peripheral nerve axons can suffer mild-to-severe damage and nerve regeneration can be slow and often incomplete (Grinsell \& Keating, 2014). Following nerve injury and subsequent sensory dysfunction, sensation returns in a specific order starting with cooling and followed by cold pain, heat pain, mechanical pain, touch, and lastly warmth (Van Boven \& Johnson, 1994). As we tested patients in the present study many months after injury, it could be that all sensations but warmth detection had returned to preinjury levels or that warmth sensation regeneration was incomplete. This finding has an important clinical implication in that patients with elevated warmth detection thresholds may be at risk of experiencing burns and subsequent injury.

The increased CPT measures at 2,000 and $250 \mathrm{~Hz}$ in cases mimic those that researchers have reported in patients with chronic pain following breast cancer surgery (Passavanti et al., 2006). Investigators have linked both traumatic injury and surgery with peripheral nerve damage, with nerves being severed, crushed, compressed, or stretched (National Institute for Neurological Disorders and Stroke, 2014). Research has found an association between increased CPT measures and demyelination of peripheral nerves (Katims, 1998; Kurozawa, Hosoda, \& Nasu, 2010), which can occur following inflammation or compression (Love, 2006) and could possibly occur 
following injury, as the pathophysiologic process that takes place at the time of traumatic injury is a complex multisystem response (Winterborn \& Cook, 2003).

While QST showed altered sensation in a subset of unmyelinated $\mathrm{C}$ fibers in the present study, CPT showed changes in myelinated $A \beta$ and $A \delta$ fibers, and patients reported pain descriptors that are often associated with neuropathies (Watson \& Dyck, 2015). A potential hypothesis to explain this disparity is that not all sensory neurons are affected equally at the time of injury. In addition to CPT, which reflects the function of afferent fibers, and QST, which reflects the function of both skin receptors and afferent fibers (Lowenstein, Jesse, \& Kenton, 2008), performing nerve conduction velocity (NCV) testing in patients with lower extremity fractures could provide vital information as to the pathophysiology of neurons following injury. The NCV test determines the extent of nerve damage by measuring the speed of conduction through a nerve using an electrical impulse (Johns Hopkins Medicine, n.d.). Abnormal results can provide insight into possible neuropathy associated with injury.

The present study had several limitations, specifically (1) the sample of participants with fractures was small and (2) we performed the testing in these participants at different time points following injury. Readers should interpret the CPT results with caution, as several participants had inconsistent responses resulting in a small sample size. In addition, some of the participants took medication for their pain, which could have affected the results. Furthermore, we had no information on the extent of nerve damage associated with the injuries; thus, it is possible that some of the participants might have had subclinical nerve injuries. We attempted to lessen the effects of this limitation by waiting to test patients until at least 6 months after injury, when one would expect the majority of subtle nerve injuries to have resolved. A future longitudinal study in which investigators perform QST, CPT, and nerve biopsies at several time points after injury would provide data about how sensory function changes as the injury heals. For all future studies, examining data from both QST and CPT testing, as we did in the present study, would increase the reliability of results.

\section{Conclusion}

The present study is the first to examine sensory nerve function at the site of injury in participants with chronic pain following lower extremity traumatic fractures using both QST and CPT. In spite of the limitations, findings of this study suggest that participants with chronic pain following lower extremity fractures may experience hypoesthesia in some aspects of somatosensation. It is important that future studies examining chronic pain following traumatic lower extremity fractures use both QST and CPT because CPT reflects the function of afferent fibers, while QST reflects the function of both skin receptors and afferent fibers (Lowenstein et al., 2008). Research contributing to the understanding of the underlying mechanisms associated with chronic pain following lower extremity fractures has the potential to markedly improve patient quality of life, aid in the development of trauma-specific practice guidelines, and decrease health-care costs associated with chronic pain.

\section{Authors' Note}

The content is solely the responsibility of the authors and does not necessarily represent the official views of the National Institutes of Health.

Permission to use copyrighted material SF-MPQ-2 (C) R. Melzack and the Initiative on Methods, Measurement, and Pain Assessment in Clinical Trials (IMMPACT), 2009. All Rights Reserved. SF-MPQ-2 contact information and permission to use: MAPI Research Trust, Lyon, France. E-mail:PROinformation@mapi-trust.org - Internet: www.mapi-trust.org STAIAD instrument (C) 1968, 1977 Charles D. Spielberger. All rights reserved in all media. Published by Mind Garden, Inc., www.mindgarden.com Beck Depression Inventory ${ }^{\mathbb{B}}$-II (BDI ${ }^{\circledR}$-II). Copyright (C) 1996 Aaron T. Beck. Used with permission of the publisher NCS Pearson, Inc. All rights reserved.

\section{Acknowledgments}

The authors thank Patty B. Casper and Joseph Larivey for their feedback on pain management in the acute care setting and Betsy Kramer for assisting in identifying eligible patients for this study.

\section{Author Contributions}

M. Griffioen contributed to conception, design, data acquisition, data analysis, and interpretation, drafted the manuscript, critically revised the manuscript, gave final approval, and agrees to be held accountable for all aspects of work, ensuring integrity and accuracy. J. Greenspan contributed to conception, design, data analysis, and interpretation, critically revised the manuscript, gave final approval, and agrees to be held accountable for all aspects of work, ensuring integrity and accuracy. M. Johantgen contributed to conception, design, data analysis, and interpretation, critically revised the manuscript, gave final approval, and agrees to be held accountable for all aspects of work, ensuring integrity and accuracy. K. Von Rueden contributed to conception, design, data acquisition, and interpretation, critically revised the manuscript, gave final approval, and agrees to be held accountable for all aspects of work, ensuring integrity and accuracy. R. O'Toole contributed to design, data analysis, and interpretation, critically revised the manuscript, gave final approval, and agrees to be held accountable for all aspects of work, ensuring integrity and accuracy. S. Dorsey contributed to conception, design, data analysis, and interpretation, critically revised the manuscript, gave final approval, and agrees to be held accountable for all aspects of work, ensuring integrity and accuracy. C. Renn contributed to conception, design, data acquisition, data analysis, and interpretation, drafted the manuscript, critically revised the manuscript, gave final approval, and agrees to be held accountable for all aspects of work, ensuring integrity and accuracy.

\section{Declaration of Conflicting Interests}

The author(s) declared no potential conflicts of interest with respect to the research, authorship, and/or publication of this article.

\section{Funding}

The author(s) disclosed receipt of the following financial support for the research, authorship, and/or publication of this article: Research 
reported in this publication was supported by the National Institute of Nursing Research of the National Institutes of Health under award number F31NR014986.

\section{References}

Castillo, R. C., MacKenzie, E. J., Wegener, S. T., \& Bosse, M. J. (2006). Prevalence of chronic pain seven years following limb threatening lower extremity trauma. Pain, 124, 321-329. doi:10.1016/j.pain.2006.04.020

Clay, F. J., Newstead, S. V., Watson, W. L., Ozanne-Smith, J., Guy, J., $\&$ McClure, R. J. (2010). Bio-psychosocial determinants of persistent pain 6 months after non-life-threatening acute orthopaedic trauma. Journal of Pain, 11, 420-430. doi:10.1016/j.jpain.2009. 12.002

Clay, F. J., Watson, W. L., Newstead, S. V., \& McClure, R. J. (2012). A systematic review of early prognostic factors for persistent pain following acute orthopedic trauma. Pain Research \& Management, 17, 35-45.

Dworkin, R. H., Turk, D. C., Revicki, D. A., Harding, G., Coyne, K. S., Peirce-Sandner, S., ... Melzack, R. (2009). Development and initial validation of an expanded and revised version of the short-form McGill Pain Questionnaire (SF-MPQ-2). Pain, 144, 35-42. doi:10.1016/j.pain.2009.02.007

Elliott, A. M., Smith, B. H., Smith, W. C., \& Chambers, W. A. (2000). Changes in chronic pain severity over time: The Chronic Pain Grade as a valid measure. Pain, 88, 303-308. Retrieved from http://www.ncbi.nlm.nih.gov/pubmed/11068118

Griffioen, M. A., Greenspan, J. D., Johantgen, M., Von Rueden, K., O'Toole, R. V., Dorsey, S. G., \& Renn, C. L. (2017). Acute pain characteristics in patients with and without chronic pain following lower extremity injury. Pain Management Nursing, 18, 33-41.

Grinsell, D., \& Keating, C. P. (2014). Peripheral nerve reconstruction after injury: A review of clinical and experimental therapies. BioMed Research International, 2014, 1-13. doi:10.1155/2014/698256

Harris, C. A., \& D'Eon, J. L. (2008). Psychometric properties of the Beck Depression Inventory - second edition (BDI-II) in individuals with chronic pain. Pain, 137, 609-622. doi:10.1016/j.pain. 2007.10.022

Jenewein, J., Moergeli, H., Wittmann, L., Büchi, S., Kraemer, B., \& Schnyder, U. (2009). Development of chronic pain following severe accidental injury. Results of a 3-year follow-up study. Journal of Psychosomatic Research, 66, 119-126. doi:10.1016/j.jpsychores.2008.07.011

Jensen, R., Hystad, T., Kvale, A., \& Baerheim, A. (2007). Quantitative sensory testing of patients with long lasting patellofemoral pain syndrome. European Journal of Pain, 11, 665-676. doi:10.1016/j. ejpain.2006.10.007

Johns Hopkins Medicine. (n.d.). Health library: Nerve conduction studies. Retrieved from http://www.hopkinsmedicine.org/healthli brary/test_procedures/neurological/nerve_conduction_velocity_ ncv_92, P07657/

Julian, L. J. (2011). Measures of anxiety: State-Trait Anxiety Inventory (STAI), Beck Anxiety Inventory (BAI), and Hospital Anxiety and Depression Scale-Anxiety (HADS-A). Arthritis Care \& Research, 63, S467-S472. doi:10.1002/acr.20561
Katims, J. J. (1998). Electrodiagnostic functional sensory evaluation of the patient with pain: A review of neuroselective current perception threshold and pain tolerance threshold. Pain Digest, 8, 219-230.

Korff, M., Ormel, J., \& Dworkin, S. F. (1992). Grading the severity of chronic pain. Pain, 50, 133-149.

Kurozawa, Y., Hosoda, T., \& Nasu, Y. (2010). Current perception threshold for assessment of the neurological components of hand-arm vibration syndrome: A review. Yonago Acta Medica, 53, 59-64.

Latremoliere, A., \& Woolf, C. J. (2009). Central sensitization: A generator of pain hypersensitivity by central neural plasticity. Journal of Pain, 10, 895-926. doi:10.1016/j.jpain. 2009.06.012

Leffler, A. S., \& Hansson, P. (2008). Painful traumatic peripheral partial nerve injury-sensory dysfunction profiles comparing outcomes of bedside examination and quantitative sensory testing. European Journal of Pain, 12, 397-402. doi:10.1016/j.ejpain. 2007.08.009

Love, S. (2006). Demyelinating diseases. Journal of Clinical Pathology, 59, 1151-1159. doi:10.1136/jcp.2005.031195

Lovejoy, T. I., Turk, D. C., \& Morasco, B. J. (2012). Evaluation of the psychometric properties of the revised short-form McGill Pain Questionnaire. Journal of Pain, 13, 1250-1257. doi:10.1016/j. jpain.2012.09.011

Lowenstein, L., Jesse, K., \& Kenton, K. (2008). Comparsion of perception threshold testing and thermal vibratory testing. Muscle and Nerve, 37, 514-517. doi:10.1002/mus.20934

National Institute for Neurological Disorders and Stroke. (2014). Peripheral neuropathy fact sheet. Retrieved from http://www.ninds. nih.gov/disorders/peripheralneuropathy/detail_peripheralneuropa thy.htm

Newcomer, K. L., Shelerud, R. A, Douglas, K. S., Larson, D. R., \& Crawford, B. J. (2010). Anxiety levels, fear-avoidance beliefs, and disability levels at baseline and at 1 year among subjects with acute and chronic low back pain. $P M \& R, 2,514-520$. doi:10.1016/ j.pmrj.2010.03.034

Passavanti, M. B., Pace, M. C., Barbarisi, A., D’Andrea, F., Grella, E., Nicoletti, G. F., \& Aurilio, C. (2006). Pain and sensory dysfunction after breast cancer surgery: Neurometer CPT evaluation. Anticancer Research, 26, 3839-3844.

Raak, R., \& Wallin, M. (2006). Thermal thresholds and catastrophizing in individuals with chronic pain after whiplash injury. Biological Research Nursing, 8, 138-146. doi:10.1177/ 1099800406291078

Rendell, M. S., Dovgan, D. J., Bergman, T. F., O’Donnell, G. P., Drobny, E. P., \& Katims, J. J. (1989). Mapping diabetic sensory neuropathy by current perception threshold testing. Diabetes Care, 12, 636-640.

Rivara, F. P., Mackenzie, E. J., Jurkovich, G. J., Nathens, A. B., Wang, J., \& Scharfstein, D. O. (2008). Prevalence of pain in patients 1 year after major trauma. Archives of Surgery, 143, 282-287. doi: 10.1001/archsurg.2007.61

Rolke, R., Baron, R., Maier, C., Tölle, T. R., Treede, R.-D., Beyer, A., ... Wasserka, B. (2006). Quantitative sensory testing in the German Research Network on Neuropathic Pain (DFNS): 
Standardized protocol and reference values. Pain, 123, 231-243. doi:10.1016/j.pain.2006.01.041

Rosenbloom, B. N., Khan, S., McCartney, C., \& Katz, J. (2013). Systematic review of persistent pain and psychological outcomes following traumatic musculoskeletal injury. Journal of Pain Research, 6, 39-51. doi:10.2147/JPR.S38878

Smarr, K. L., \& Keefer, A. L. (2011). Measures of depression and depressive symptoms: Beck Depression Inventory-II (BDI-II), Center for Epidemiologic Studies Depression Scale (CES-D), Geriatric Depression Scale (GDS), Hospital Anxiety and Depression Scale (HADS), and Patient Health Questionna. Arthritis Care \& Research, 63, S454-S466. doi:10.1002/acr.20556

Stålnacke, B.-M. (2011). Life satisfaction in patients with chronic pain - relation to pain intensity, disability, and psychological factors. Neuropsychiatric Disease and Treatment, 7, 683-689. doi:10. 2147/NDT.S25321

Starkweather, A. R., Lyon, D. E., Kinser, P., Heineman, A., Sturgill, J. L., Deng, X., ... Dorsey, S. G. (2016). Comparison of low back pain recovery and persistence: A descriptive study of characteristics at pain onset. Biological Research for Nursing, 18, 401-410. doi:10.1177/1099800416631819
Trauma.org. (2007). Trauma scoring: Abbreviated Injury Scale. Retrieved January 22, 2015, from http://www.trauma.org/ archive/scores/ais.html

Van Boven, R. W., \& Johnson, K. O. (1994). A psychophysical study of the mechanisims of sensory recovery following nerve injury in humans. Pain, 117, 149-167.

Watson, J. C., \& Dyck, P. J. B. (2015). Peripheral neuropathy: A practical approach to diagnosis and symptom management. Mayo Clinic Proceedings, 90, 940-951. doi:10.1016/j.mayocp. 2015.05.004

Williamson, O. D., Gabbe, B. J., Cameron, P. A., Edwards, E. R., \& Richardson, M. D. (2009). Predictors of moderate or severe pain 6 months after orthopaedic injury: A prospective cohort study. Journal of Orthopaedic Trauma, 23, 139-144. doi:10.1097/BOT. 0b013e3181962e 29

Winterborn, R. J., \& Cook, T. A. (2003). The pathophysiology of severe trauma. Surgery, 21, 240a-240e. doi:10.1383/surg.21.9. 240.16923

Woolf, C. J. A. (2011). Central sensitization: Implications for the diagnosis and treatment of pain. Pain, 152, 1-31. doi:10.1016/ j.pain.2010.09.030.Central 